\title{
Damage detection from the variation of parameter matrices estimated by incomplete FRF data
}

\author{
Salam Rahmatalla ${ }^{1}$, Hee-Chang Eun*2 and Eun-Taik Lee ${ }^{3}$ \\ ${ }^{1}$ Faculty of Department of Civil and Environmental Engineering, University of lowa, lowa City, USA \\ ${ }^{2}$ Faculty of Department of Architectural Engineering, Kangwon National University, Chuncheon, Korea \\ ${ }^{3}$ Faculty of Department of Architectural Engineering, Chung-Ang University, Seoul, Korea
}

(Received December 17, 2010, Revised July 9, 2011, Accepted December 20, 2011)

\begin{abstract}
It is not easy to experimentally obtain the FRF (Frequency Response Function) matrix corresponding to a full set of DOFs (degrees of freedom) for a dynamic system. Utilizing FRF data measured at specific positions, with DOFs less than that of the system, as constraints to describe a damaged system, this study identifies parameter matrices such as mass, stiffness and damping matrices of the system, and provides a damage identification method from their variations. The proposed parameter identification method is compared to Lee and Kim's method and Fritzen's method. The validity of the proposed damage identification method is illustrated in a simple dynamic system.
\end{abstract}

Keywords: frequency response function; dynamic stiffness matrix; receptance; damping matrix; minimization.

\section{Introduction}

An accurate dynamic finite element model of a structure is very important for structural design and analysis. The modes of vibration and frequency response of the finite element model are compared to experimental measurements to test its accuracy. If a discrepancy between the two is found, the analytical model should be modified to satisfy the experimental measurements, and then the model should be updated for subsequent simulation and design studies.

An FRF matrix of the full set of DOFs measured by experiments is used to predict parameter matrices of the dynamic system. The FRFs have been used directly to update condensed analytical models for obtaining the proper modal model. A single FRF measured at several frequencies, along with a correlated analytical model of the structure in its original state, is used for updating structural parameters. There have been many attempts to update the unknown physical parameters directly from the FRFs (Mottershead and Stanway 1986). It has been reported (Lee and Shin 2002) that FRF data provide more information than modal data, as the latter are extracted from a very limited frequency range related to resonance. Friswell and Penny (1990) proposed an approach to reduce the model order so that the stated estimation process reduces to a least squares problem based on the FRFs. Fanning and Carden (2004) presented a method for detecting added mass in structural systems from a single FRF measured at several frequencies in an identification algorithm. Cha and

*Corresponding author, Professor, E-mail: heechang@kangwon.ac.kr 
Tuck-Lee (2000) developed approaches to update mass and stiffness matrices based on two sets of measured frequency response data. Kwon and Lin (2004) proposed a frequency selection method for efficient FRF-based model updating. Lin and Zhu (2006) developed model updating methods to identify mass, stiffness, and damping matrices of a damaged dynamic system based on the FRF method. Phani and Woodhouse (2007) identified parameter matrices of viscous damping models based on measured FRFs. They compared matrix methods using a measured FRF matrix and modal methods using modal parameters. Inverting the FRF matrix to the dynamic stiffness matrix and comparing their real and imaginary parts with parameter matrices, Lee and Kim (2001) identified damping characteristics of the system in matrix forms directly from its measured FRFs. Fritzen (1986) proposed an analytical method to describe the parameter matrices by minimizing the error of a dynamic stiffness matrix and its inverse, and an identity matrix.

The spatially sampled field measured during experiments has been an active area of research for many years. Generally, the measured data are less than the DOFs of the system. The analysis is performed by reducing the DOFs of the system or by expanding the measured data. Most expansion techniques involve the use of the finite element model as a mechanism to complete the unmeasured DOFs from the experimental model. Guyan (1965) presented a transformation matrix to establish the relationship between the expanded eigenvector and the measured components. The Improved Reduced System (1989) is an improvement on the static reduction method and provides a perturbation to the transformation from the static case by including the inertia terms as pseudo static forces. Using mass-normalized real mode shapes determined by finite element models, O'Callahan and Avitable (1989) proposed the System Equivalent Reduction-Expansion Process (SEREP). Based on a dynamic modal expansion that minimizes the residual error in the eigenvalue equation for each measured mode, Kenneth and Francois (2000) presented an algorithm for expanding measured mode shapes obtained from modal testing to the full set of DOFs of a corresponding finite element model. Ewins (2000) presented some definitions and a very concise summary of each of the major algorithms for updating the problem, along with discussions of how and when each of these algorithms might be used.

The number of measured FRF data for system identification can also be less than the number of DOFs in the system, and the measured data should be increased for obtaining more accurate parameter matrices. The incomplete measured FRF data explain a part of the dynamic response, and they are expanded to the size of the analytical model for identifying the parameter matrices of the entire system instead of reducing DOFs of the system. The expanded FRF matrix provides some basic information about detecting damage in mechanical systems. Avitabile and O'Callahan (2001) provided a method to expand measured translational FRFs using the measured modes of the system along with the residual modes from the modal parameter estimation process. They (Avitabile and O'Callahan 2003) presented the general model reduction methodology, the mode shape expansion, the residual mode expansion, and the FRF expansion and developed the FRFs involving translational to rotational DOFs as well as the rotational to rotational DOF. Phani and Seshia (2004) expanded the FRF matrix of the non-proportionally damped system in terms of the FRF matrix of the proportionally damped system using a matrix perturbation method.

Although an initial theoretical model should be refined, corrected, or updated based on measured data from the actual structure, current expansion methods are limited to the methods to expand the experimentally measured DOFs over all DOFs under the assumption that the dynamic properties of the initial system are invariant. Using the measured FRFs as constraint conditions to describe the dynamic response, this study presents an analytical method to expand the measured FRFs to a full set of FRF matrix corresponding to the entire system, the identification method of parameter matrices 
from the expanded FRF matrix and the damage identification method from the variation in the parameter matrices. The proposed parameter identification method is compared with Lee and Kim's method (2001) and Fritzen's method (1986). The dynamic system considered in this study includes a non-proportional damping matrix for the system identification. It is illustrated that the proposed method can be used to detect low-level damage.

\section{Expansion of FRF matrix using incomplete FRF data}

Dynamic responses are expressed in the time domain and frequency domain. For linear systems there is little loss of information going from the time domain to the frequency domain. The FRFs in the frequency domain are measured instead of displacement and force individually. The dynamic behaviour of a structure that is assumed to be linear and approximately discretized for $n$ DOFs can be described by the equations of motion as

$$
\mathbf{M u ̈}+\mathbf{C} \dot{\mathbf{u}}+\mathbf{K u}=\mathbf{f}(t)
$$

where $\mathbf{M}$ and $\mathbf{K}$ denote the $n \times n$ mass and stiffness matrices, $\mathbf{u}=\left[\begin{array}{llll}u_{1} & u_{2} & \ldots & u_{n}\end{array}\right]^{T}, \mathbf{C} \in R^{n \times n}$ is the damping matrix, and $\mathbf{f}(t)$ is the $n \times 1$ excitation vector.

It is important to establish the relationships between FRF and modal parameters for successful modal testing. Inserting $\mathbf{u}=\mathbf{U} e^{i \Omega t}$ and $\mathbf{f}=\mathbf{F} e^{i \Omega t}$ into Eq. (1) and expressing it in the frequency domain, it follows that

$$
\mathbf{D}(\Omega) \mathbf{U}(\Omega)=\mathbf{F}(\Omega)
$$

where the dynamic stiffness matrix $\mathbf{D}$ is written by

$$
\mathbf{D}(\Omega)=\mathbf{K}-\Omega^{2} \mathbf{M}+i \Omega \mathbf{C}
$$

where $i=\sqrt{-1}, \Omega$ denotes the excitation frequency, $\mathbf{F}(\Omega)=\left[\begin{array}{llll}F_{1} & F_{2} & \ldots & F_{n}\end{array}\right]^{T}$ and $\mathbf{U}(\Omega)=\left[\begin{array}{llll}U_{1} & U_{2} & \ldots & U_{n}\end{array}\right]^{T}$ represents the Fourier transform of the force and response vectors $\mathbf{f}$ and $\mathbf{u}$, respectively. Eq. (2) is valid for an excitation frequency $\Omega$. The dynamic stiffness matrix is a positive definite matrix because it is espressed by the consistent mass, stiffness and damping matrices. Defining the FRF matrix $\hat{\mathbf{H}}$,

$$
\hat{\mathbf{H}} \equiv\left[\mathbf{K}-\Omega^{2} \mathbf{M}+i \Omega \mathbf{C}\right]^{-1}
$$

where $\hat{\mathbf{H}}(\Omega)=\left[\begin{array}{cccc}\hat{H}_{11} & \hat{H}_{12} & \cdots & \hat{H}_{1 n} \\ \hat{H}_{21} & \hat{H}_{22} & \cdots & \hat{H}_{2 n} \\ \vdots & \vdots & \ddots & \vdots \\ \hat{H}_{n 1} & \hat{H}_{n 2} & \cdots & \hat{H}_{n n}\end{array}\right], \quad \hat{H}_{i j}$ denotes the displacement response measured at location $i$ due to the unit impulse force input at location $j$.

Assuming that the initial system is unexpectedly damaged, the dynamic response of the system in the frequency domain does not satisfy Eq. (2). As a result of the damage, the physical parameter matrices or the dynamic stiffness matrix should be changed to describe the damaged system. To 
update the parameter matrices of a full set of DOFs, it is necessary to expand the measured data. This section derives an analytical method to expand the measured FRF data to a full set of FRF matrix.

Assume that the FRF data of the damaged system were measured at $m$ different positions. The measured $m \times n$ FRF matrix and the response vector have the relationship of

$$
\mathbf{A} \mathbf{U}_{d}=\mathbf{G F}
$$

where $\mathbf{A}$ is an $m \times n$ Boolean matrix to define the measured locations, $\mathbf{U}_{d}$ represents the updated displacement vector, including the measured displacement vector, $\mathbf{U}_{d}=\left[\begin{array}{l}\mathbf{U}_{d, \mathrm{~m}} \\ \mathbf{U}_{d, u}\end{array}\right]_{n \times 1}$, and $\mathbf{F}=\left[\begin{array}{l}\mathbf{F}_{\mathrm{m}} \\ \mathbf{F}_{u}\end{array}\right]_{n \times 1}$. The subscripts $m$ and $u$ represent the measured and unmeasured DOFs, respectively. $\mathbf{G}$ is $m \times n$ coefficient matrix representing the measured FRF data, $\mathbf{U}_{d, m}$ and $\mathbf{F}_{m}$ are $m \times 1$ displacement and force vectors corresponding to the measured locations, and $\mathbf{U}_{d, u}$ and $\mathbf{F}_{u}$ are $(n-m) \times 1$ displacement and force vectors corresponding to the unmeasured locations. The measured FRF matrix relation of Eq. (5) can be regarded as constraint conditions to describe the damaged system. The newly updated dynamic equation subjected to linear constraints can be derived using the method shown in the appendix. It is derived from the initial dynamic equation in the frequency domain of Eq. (2) and the constraint equations of Eq. (5).

Based on the method introduced in the appendix, the updated response vector $\mathbf{U}_{d}$ is derived as

$$
\mathbf{U}_{d}=\mathbf{U}+\Delta \mathbf{U}
$$

where $\mathbf{U}=\mathbf{D}^{-1} \mathbf{F}=\hat{\mathbf{H}} \mathbf{F}$ and

$$
\Delta \mathbf{U}=\left[\mathbf{D}^{-1 / 2}\left(\mathbf{A D}^{-1 / 2}\right)^{+}\left(\mathbf{G}-\mathbf{A} \mathbf{D}^{-1}\right)\right] \mathbf{F}=\left[\hat{\mathbf{H}}^{1 / 2}\left(\mathbf{A} \hat{\mathbf{H}}^{1 / 2}\right)^{+}(\mathbf{G}-\mathbf{A} \hat{\mathbf{H}})\right] \mathbf{F}
$$

where the superscript ' + ' indicates the Moore-Penrose inverse. $\Delta \mathbf{U}$ of Eq. (7) indicates the variation in the displacement caused by the damage and it is deeply related with the variation in the parameter matrices, $\mathbf{M}, \mathbf{C}$ and $\mathbf{K}$. It means that the damage can be evaluated by investigating the variation in the parameter matrices. Pre-multiplying both sides of Eq. (6) by the matrix D, it becomes

$$
\mathbf{D} \mathbf{U}_{d}=\mathbf{F}+\mathbf{D}^{1 / 2}\left(\mathbf{A D}^{-1 / 2}\right)^{+}\left(\mathbf{G}-\mathbf{A} \mathbf{D}^{-1}\right) \mathbf{F}
$$

The second term in the right-hand side of Eq. (8) indicates the additional force vector required for obtaining the measured FRF data in the frequency domain, and the response of the damaged system can be described by the additional action of the calculated forces

$$
\mathbf{F}^{c}=\mathbf{D}^{1 / 2}\left(\mathbf{A D}^{-1 / 2}\right)^{+}\left(\mathbf{G}-\mathbf{A} \mathbf{D}^{-1}\right) \mathbf{F}=\hat{\mathbf{H}}^{-1 / 2}\left(\mathbf{A} \hat{\mathbf{H}}^{1 / 2}\right)^{+}(\mathbf{G}-\mathbf{A} \hat{\mathbf{H}}) \mathbf{F}
$$

The coefficient matrix of the force $\mathbf{F}$ in Eq. (9) represents the variation in the FRF matrix, $\Delta \mathbf{H}$, due to the mechanical change of the dynamic system. This equation can be utilized in determining the FRF matrix of the dynamic system subjected to linear constraints. Thus, the updated FRF matrix $\mathbf{H}$ can be predicted by

$$
\mathbf{H}=\hat{\mathbf{H}}+\Delta \mathbf{H}
$$




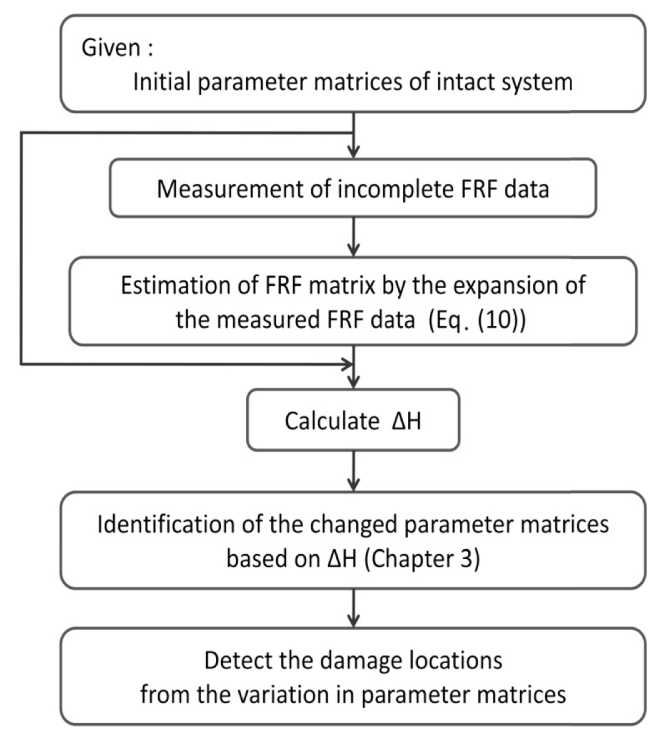

Fig. 1 Flow chart of damage detection process

where $\Delta \mathbf{H}=\hat{\mathbf{H}}^{-1 / 2}\left(\mathbf{A} \hat{\mathbf{H}}^{1 / 2}\right)^{+}(\mathbf{G}-\mathbf{A} \hat{\mathbf{H}})$.

Eq. (10) represents the entire FRF matrix of the damaged dynamic system obtained from the expansion of incomplete FRF data. The $\Delta \mathbf{H}$ indicates the variation in FRF matrix and provides the physical information on the damaged system. The variation is decomposed to the variation in the parameter matrices by proper system identification methods. The damage locations should be detected by comparing the variations in the parameter matrices. The process of damage detection presented in this study is summarized in Fig. 1.

In the following, we provide the analytical method to identify the parameter matrices from the updated FRF matrix with the object of detecting the damage. And the proposed parameter identification method is compared with Lee and Kim's method and Fritzen's method.

\section{Identification method of parameter matrices of damped dynamic system}

This section introduces Lee and Kim's method and Fritzen's method, derives an analytical method to identify parameter matrices from FRF data and compares the results of the three methods.

\subsection{Lee and Kim's method}

The FRF matrix $\mathbf{H}$ is composed of real and imaginary parts. Expressing the relation between the dynamic stiffness matrix and the FRF matrix in terms of real and imaginary parts, it follows that

$$
\mathbf{H}^{-1}=\mathbf{D}=\operatorname{Re}(\mathbf{D}(\Omega))+i \operatorname{Im}(\mathbf{D}(\Omega))
$$

where $R e$ and $I m$ indicate the real and imaginary parts of the complex, respectively, and $i=\sqrt{-1}$. The dynamic stiffness matrix $\mathbf{D}$ is obtained by taking the inverse of the FRF matrix of complex values. 
From Eqs. (4) and (11), the following relationship between the parameter matrices and the FRFs is established.

$$
\begin{gathered}
{\left[\begin{array}{ll}
\mathbf{I} & -\Omega^{2} \mathbf{I}
\end{array}\right]\left[\begin{array}{l}
\mathbf{K} \\
\mathbf{M}
\end{array}\right]=\operatorname{Re}(\mathbf{D}(\Omega))} \\
\Omega \mathbf{C}=\operatorname{Im}(\mathbf{D}(\Omega))
\end{gathered}
$$

Assuming $m$ distinct frequency response data points are experimentally measured at excitation frequencies denoted by $\left[\Omega_{1} \Omega_{2} \ldots \Omega_{m}\right]$, Eqs. (12) are expanded as

$$
\begin{gathered}
{\left[\begin{array}{cc}
\mathbf{I} & -\Omega_{1}^{2} \mathbf{I} \\
\mathbf{I} & -\Omega_{2}^{2} \mathbf{I} \\
\vdots & \vdots \\
\mathbf{I} & -\Omega_{m}^{2} \mathbf{I}
\end{array}\right]_{(n \times m) \times 2 n} \quad\left[\begin{array}{c}
\mathbf{K} \\
\mathbf{M}
\end{array}\right]_{2 n \times n}=\left[\begin{array}{c}
\operatorname{Re}\left(\mathbf{D}\left(\Omega_{1}\right)\right) \\
\operatorname{Re}\left(\mathbf{D}\left(\Omega_{2}\right)\right) \\
\vdots \\
\operatorname{Re}\left(\mathbf{D}\left(\Omega_{m}\right)\right)
\end{array}\right]_{(n \times m) \times n}} \\
{\left[\begin{array}{c}
\Omega_{1} \\
\Omega_{2} \\
\vdots \\
\Omega_{m}
\end{array}\right]_{(n \times m) \times n} \quad \mathbf{C}_{n \times n}=\left[\begin{array}{c}
\operatorname{Im}\left(\mathbf{D}\left(\Omega_{1}\right)\right) \\
\operatorname{Im}\left(\mathbf{D}\left(\Omega_{2}\right)\right) \\
\vdots \\
\operatorname{Im}\left(\mathbf{D}\left(\Omega_{2}\right)\right)
\end{array}\right]_{(n \times m) \times n}}
\end{gathered}
$$

The parameter matrices are estimated by calculating the Moore-Penrose inverse of the coefficient matrices in Eqs. (13). This method can identify all three parameter matrices by using the data of at least two frequency points. It is observed from Eqs. (13) that this method requires the inverse of the entire FRF matrix of experimentally measured complex values to obtain the dynamic stiffness matrix, unlike the proposed method and the Fritzen's method.

\subsection{Fritzen's method}

This method estimates all three parameter matrices simultaneously. Post-multiplying both sides of $\mathbf{H}=\left(\mathbf{K}-\Omega^{2} \mathbf{M}+i \Omega \mathbf{C}\right)^{-1}$ by $\left(\mathbf{K}-\Omega^{2} \mathbf{M}+i \Omega \mathbf{C}\right)$ and arranging the result yields

$$
\mathbf{H}\left(\mathbf{K}-\Omega^{2} \mathbf{M}+i \Omega \mathbf{C}\right)=\mathbf{I}
$$

Eq. (14) can be also written, divided into real and imaginary parts, as

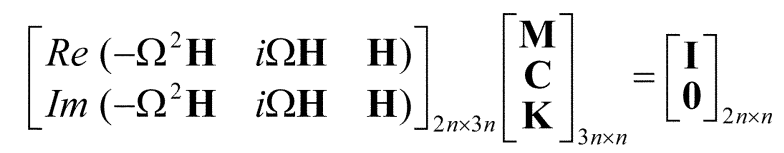

Fritzen identified the parameter matrices from Eq. (15) by an iteration process. Expanding Eq. (15) in a specific frequency range of $\left[\begin{array}{lllll}\Omega_{1} & \Omega_{2} & \ldots & \Omega_{m-1} & \Omega_{m}\end{array}\right]^{T}$, it is written as

$$
\mathbf{X}\left[\begin{array}{l}
\mathbf{M} \\
\mathbf{C} \\
\mathbf{K}
\end{array}\right]=\mathbf{Y}
$$




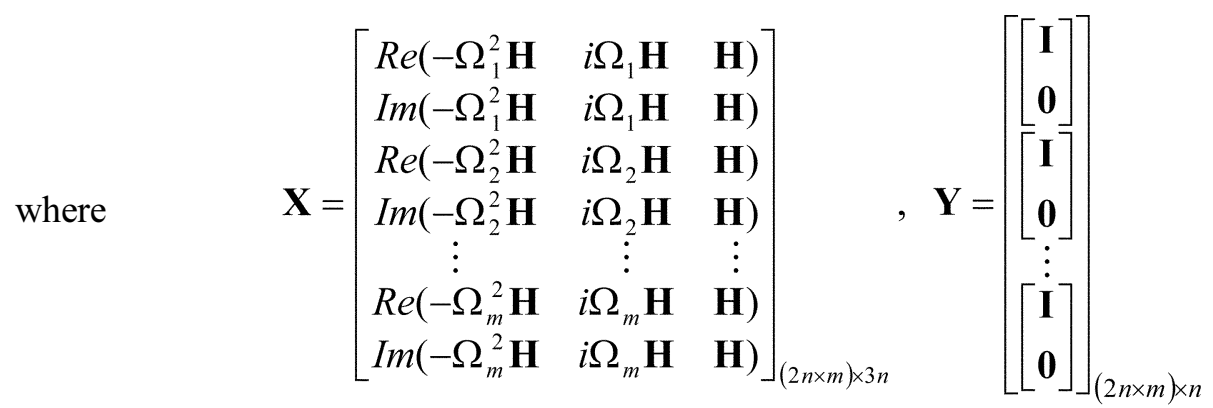

Thus, all three parameter matrices can be derived without any iteration process by utilizing the MoorePenrose inverse of the coefficient matrices in Eq. (16), as

$$
\left[\begin{array}{l}
\mathbf{M} \\
\mathbf{C} \\
\mathbf{K}
\end{array}\right]=\mathbf{X}^{+} \mathbf{Y}
$$

It is shown that this method requires more calculations than the proposed method and the Lee \& Kim's method because it calculates the Moore-Penrose inverse of the coefficient matrices to obtain all three parameter matrices simultaneously. This method, however, does not require the inverse of the entire FRF matrix including complex values.

\subsection{Identification of parameter matrices}

The FRF matrix corresponding to the full set of DOFs can be written as

$$
\mathbf{H}=\mathbf{H}_{r e}+i \mathbf{H}_{i m}
$$

where $\mathbf{H}_{r e}$ and $\mathbf{H}_{i m}$ denote the real and imaginary parts of the FRF matrix. Substituting Eq. (18) into Eq. (4) yields

$$
\left(\mathbf{H}_{r e}+i \mathbf{H}_{i m}\right)\left(\mathbf{K}+i \Omega \mathbf{C}-\Omega^{2} \mathbf{M}\right)=\mathbf{I}
$$

Arranging Eq. (19) into real and imaginary parts, it can be expressed as

$$
\left[\begin{array}{cc}
\mathbf{H}_{r e} & -\mathbf{H}_{i m} \\
\mathbf{H}_{i m} & \mathbf{H}_{r e}
\end{array}\right]\left[\begin{array}{c}
\mathbf{K}-\Omega^{2} \mathbf{M} \\
\Omega \mathbf{C}
\end{array}\right]=\left[\begin{array}{l}
\mathbf{I} \\
\mathbf{0}
\end{array}\right]
$$

Solving the first and second equations of Eq. (20) with respect to $\left(\mathbf{K}-\Omega^{2} \mathbf{M}\right)$ and $\Omega \mathbf{C}$, respectively, yields

$$
\begin{gathered}
\left(\mathbf{K}-\Omega^{2} \mathbf{M}\right)=\mathbf{H}_{r e}^{-1}\left(\mathbf{I}+\mathbf{H}_{i m}(\Omega \mathbf{C})\right) \\
\Omega \mathbf{C}=-\mathbf{H}_{r e}^{-1} \mathbf{H}_{i m}\left(\mathbf{K}-\Omega^{2} \mathbf{M}\right)
\end{gathered}
$$

Substituting Eq. (21(a)) into Eq. (21(b)) and arranging the result yields 


$$
\Omega \mathbf{C}=-\mathbf{H}_{r e}^{-1}\left(\mathbf{H}_{r e} \mathbf{H}_{i m}^{-1}+\mathbf{H}_{r e}^{-1} \mathbf{H}_{i m}\right)^{-1}
$$

Utilizing Eq. (22) in Eq. (21(a)) and arranging it with respect to the stiffness and mass matrices results in

$$
\mathbf{K}-\Omega^{2} \mathbf{M}=\mathbf{H}_{i m}^{-1}\left(\mathbf{H}_{r e} \mathbf{H}_{i m}^{-1}+\mathbf{H}_{r e}^{-1} \mathbf{H}_{i m}\right)^{-1}
$$

Expanding Eqs. (22) and (23) in a specific frequency range of $\left[\begin{array}{lll}\Omega_{1} & \Omega_{2} \ldots \Omega_{m-1} & \Omega_{m}\end{array}\right]^{T}$, it is modified as

$$
\begin{gathered}
{\left[\begin{array}{c}
\Omega_{1} \mathbf{I} \\
\Omega_{2} \mathbf{I} \\
\vdots \\
\Omega_{n} \mathbf{I}
\end{array}\right] \mathbf{C}=\left[\begin{array}{c}
-\mathbf{H}_{r e}^{-1}\left(\Omega_{1}\right)\left(\mathbf{H}_{r e}\left(\Omega_{1}\right) \mathbf{H}_{i m}^{-1}\left(\Omega_{1}\right)+\mathbf{H}_{r e}^{-1}\left(\Omega_{1}\right) \mathbf{H}_{i m}\left(\Omega_{1}\right)\right)^{-1} \\
-\mathbf{H}_{r e}^{-1}\left(\Omega_{2}\right)\left(\mathbf{H}_{r e}\left(\Omega_{2}\right) \mathbf{H}_{i m}^{-1}\left(\Omega_{2}\right)+\mathbf{H}_{r e}^{-1}\left(\Omega_{2}\right) \mathbf{H}_{i m}\left(\Omega_{2}\right)\right)^{-1} \\
\vdots \\
-\mathbf{H}_{r e}^{-1}\left(\Omega_{n}\right)\left(\mathbf{H}_{r e}\left(\Omega_{n}\right) \mathbf{H}_{i m}^{-1}\left(\Omega^{\prime}\right)+\mathbf{H}_{r e}^{-1}\left(\Omega_{n}\right) \mathbf{H}_{i m}\left(\Omega_{n}\right)\right)^{-1}
\end{array}\right]} \\
{\left[\begin{array}{cc}
\mathbf{I} & -\Omega_{1}^{2} \mathbf{I} \\
\mathbf{I} & -\Omega_{2}^{2} \mathbf{I} \\
\vdots & \vdots \\
\mathbf{I} & -\Omega_{n}^{2} \mathbf{I}
\end{array}\right]\left[\begin{array}{c}
\mathbf{K} \\
\mathbf{M}
\end{array}\right]=\left[\begin{array}{c}
\mathbf{H}_{i m}^{-1}\left(\Omega_{1}\right)\left(\mathbf{H}_{r e}\left(\Omega_{1}\right) \mathbf{H}_{i m}^{-1}\left(\Omega_{1}\right)+\mathbf{H}_{r e}^{-1}\left(\Omega_{1}\right) \mathbf{H}_{i m}\left(\Omega_{1}\right)\right)^{-1} \\
\mathbf{H}_{i m}^{-1}\left(\Omega_{2}\right)\left(\mathbf{H}_{r e}\left(\Omega_{2}\right) \mathbf{H}_{i m}^{-1}\left(\Omega_{2}\right)+\mathbf{H}_{r e}^{-1}\left(\Omega_{2}\right) \mathbf{H}_{i m}\left(\Omega_{2}\right)\right)^{-1} \\
\vdots \\
\mathbf{H}_{i m}^{-1}\left(\Omega_{n}\right)\left(\mathbf{H}_{r e}\left(\Omega_{n}\right) \mathbf{H}_{i m}^{-1}(\Omega)+\mathbf{H}_{r e}^{-1}\left(\Omega_{n}\right) \mathbf{H}_{i m}\left(\Omega_{n}\right)\right)^{-1}
\end{array}\right]}
\end{gathered}
$$

The updated parameter matrices can be written as

$$
\begin{gathered}
\mathbf{C}=\left[\begin{array}{c}
\Omega_{1} \mathbf{I} \\
\Omega_{2} \mathbf{I} \\
\vdots \\
\Omega_{n} \mathbf{I}
\end{array}\right]^{+}\left[\begin{array}{c}
-\mathbf{H}_{r e}^{-1}\left(\Omega_{1}\right)\left(\mathbf{H}_{r e}\left(\Omega_{1}\right) \mathbf{H}_{i m}^{-1}\left(\Omega_{1}\right)+\mathbf{H}_{r e}^{-1}\left(\Omega_{1}\right) \mathbf{H}_{i m}\left(\Omega_{1}\right)\right)^{-1} \\
-\mathbf{H}_{r e}^{-1}\left(\Omega_{2}\right)\left(\mathbf{H}_{r e}\left(\Omega_{2}\right) \mathbf{H}_{i m}^{-1}\left(\Omega_{2}\right)+\mathbf{H}_{r e}^{-1}\left(\Omega_{2}\right) \mathbf{H}_{i m}\left(\Omega_{2}\right)\right)^{-1} \\
\vdots \\
-\mathbf{H}_{r e}^{-1}\left(\Omega_{n}\right)\left(\mathbf{H}_{r e}\left(\Omega_{n}\right) \mathbf{H}_{i m}^{-1}(\Omega)+\mathbf{H}_{r e}^{-1}\left(\Omega_{n}\right) \mathbf{H}_{i m}\left(\Omega_{n}\right)\right)^{-1}
\end{array}\right] \\
{\left[\begin{array}{c}
\mathbf{K} \\
\mathbf{M}
\end{array}\right]=\left[\begin{array}{cc}
\mathbf{I} & -\Omega_{1}^{2} \mathbf{I} \\
\mathbf{I} & -\Omega_{2}^{2} \mathbf{I} \\
\vdots & \vdots \\
\mathbf{I} & -\Omega_{n}^{2} \mathbf{I}
\end{array}\right]\left[\begin{array}{c}
\mathbf{H}_{i m}^{-1}\left(\Omega_{1}\right)\left(\mathbf{H}_{r e}\left(\Omega_{1}\right) \mathbf{H}_{i m}^{-1}\left(\Omega_{1}\right)+\mathbf{H}_{r e}^{-1}\left(\Omega_{1}\right) \mathbf{H}_{i m}\left(\Omega_{1}\right)\right)^{-1} \\
\mathbf{H}_{i m}^{-1}\left(\Omega_{2}\right)\left(\mathbf{H}_{r e}\left(\Omega_{2}\right) \mathbf{H}_{i m}^{-1}\left(\Omega_{2}\right)+\mathbf{H}_{r e}^{-1}\left(\Omega_{2}\right) \mathbf{H}_{i m}\left(\Omega_{2}\right)\right)^{-1} \\
\vdots \\
\mathbf{H}_{i m}^{-1}\left(\Omega_{n}\right)\left(\mathbf{H}_{r e}\left(\Omega_{n}\right) \mathbf{H}_{i m}^{-1}\left(\Omega_{)}\right) \mathbf{H}_{r e}^{-1}\left(\Omega_{n}\right) \mathbf{H}_{i m}\left(\Omega_{n}\right)\right)^{-1}
\end{array}\right]}
\end{gathered}
$$

This method requires the inverse of each coefficient matrix of the real and imaginary parts instead of the inverse of the entire FRF matrix of complex numbers. And this method does not require the calculation of the Moore-Penrose inverse to obtain all three parameter matrices simultaneously.

Eqs. (13), (17), and (25) provide the information to describe the parameter matrices, although they take different forms. The damage detection method to present in this study begins with a limited number of measured data and parameter matrices of the intact structure, expands them to the full set of FRF matrix, identifies the updated parameter matrices and then detects the damage by comparing the variation in the parameter matrices. 
In the following application, we investigate the validity of the proposed damage identification method from the variation in the parameter matrices and compare the derived parameter identification method with the other methods.

\section{Application of damage detection method}

As an application, we consider a dynamic system of 40 DOFs shown in Fig. 2. The numerical values of physical parameters are $m_{1}=m_{2}=\cdots=m_{40}=4 \mathrm{~kg}$ and $k_{1}=k_{2} \cdots=k_{40}=1.2 \times 10^{3} \mathrm{~N} / \mathrm{mm}$. The viscous damping coefficient takes $\alpha=\beta=0.0001$ for the Rayleigh's damping where $\alpha$ and $\beta$ represent the damping constants for viscous damping. This application considers a non-proportional damping system by reducing the distribution of the stiffness to the damping matrix by $50 \%$ in the $37^{\text {th }}$ element. This numerical application established the stiffnesses of seven elements as $0.9 k_{8}, 0.95 k_{11}$, $0.85 k_{17}, 0.96 k_{23}, 0.9 k_{28}, 0.95 k_{33}$, and $0.93 k_{37}$, where the subscripts correspond to damage elements. The damage rate was selected in the range of $4 \%-15 \%$ of the initial stiffness.

The damping matrix in this system is closely related to the stiffness and mass matrices because of the assumption of the Rayleigh's damping. For this numerical application, we assume that the FRF data corresponding to only the first 15 DOFs out of 40 DOFs of the dynamic system were measured. The simulated incomplete data are treated as experimental data by generating a damped model with non-proportional viscous damping. Using the measured data and the initial parameter matrices into Eq. (10), we obtain the updated FRF matrix of the system. This numerical application begins with the dynamic system of noise-free FRF data in the range of $0.01-40 \mathrm{~Hz}$ in steps of $0.02 \mathrm{~Hz}$.

Fig. 3 represents the FRF curves and discrepancy rate between the actual FRF and the analytically identified FRFs estimated at measured and unmeasured nodes. The discrepancy rate is defined as

$$
\frac{\log _{10}(\mathbf{H})_{a c t .}-\log _{10}(\mathbf{H})_{c a l} .}{\log _{10}(\mathbf{H})_{a c t}} \times 100(\%)
$$

It is observed that the maximum discrepancy in receptance magnitude is located at the antiresonance frequencies, and the analytical methods provided by Lee and Kim, Fritzen, and this study yield the same FRF curves as those at the measured locations. But the FRF curves at the unmeasured positions exhibit lower discrepancy rates than $10 \%$. This observation indicates that the proposed expansion method will gradually approach the actual FRF matrix with the increase in the measured DOFs.

The variation in FRF matrix due to damage is calculated by Eq. (10) and provides the variation in parameter matrices by the proposed system identification method. The variation in parameter matrices is utilized as an index to evaluate the damage. Fig. 4 represents the discrepancy in the

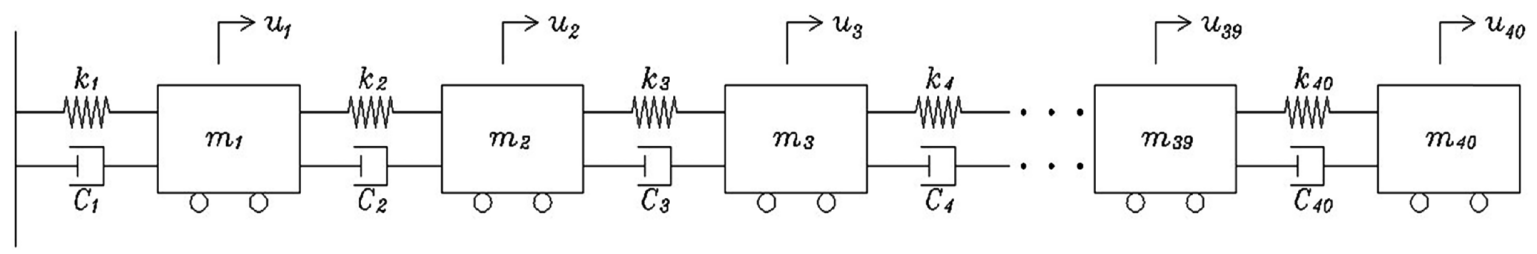

Fig. 2 A dynamic system of 40 DOFs 

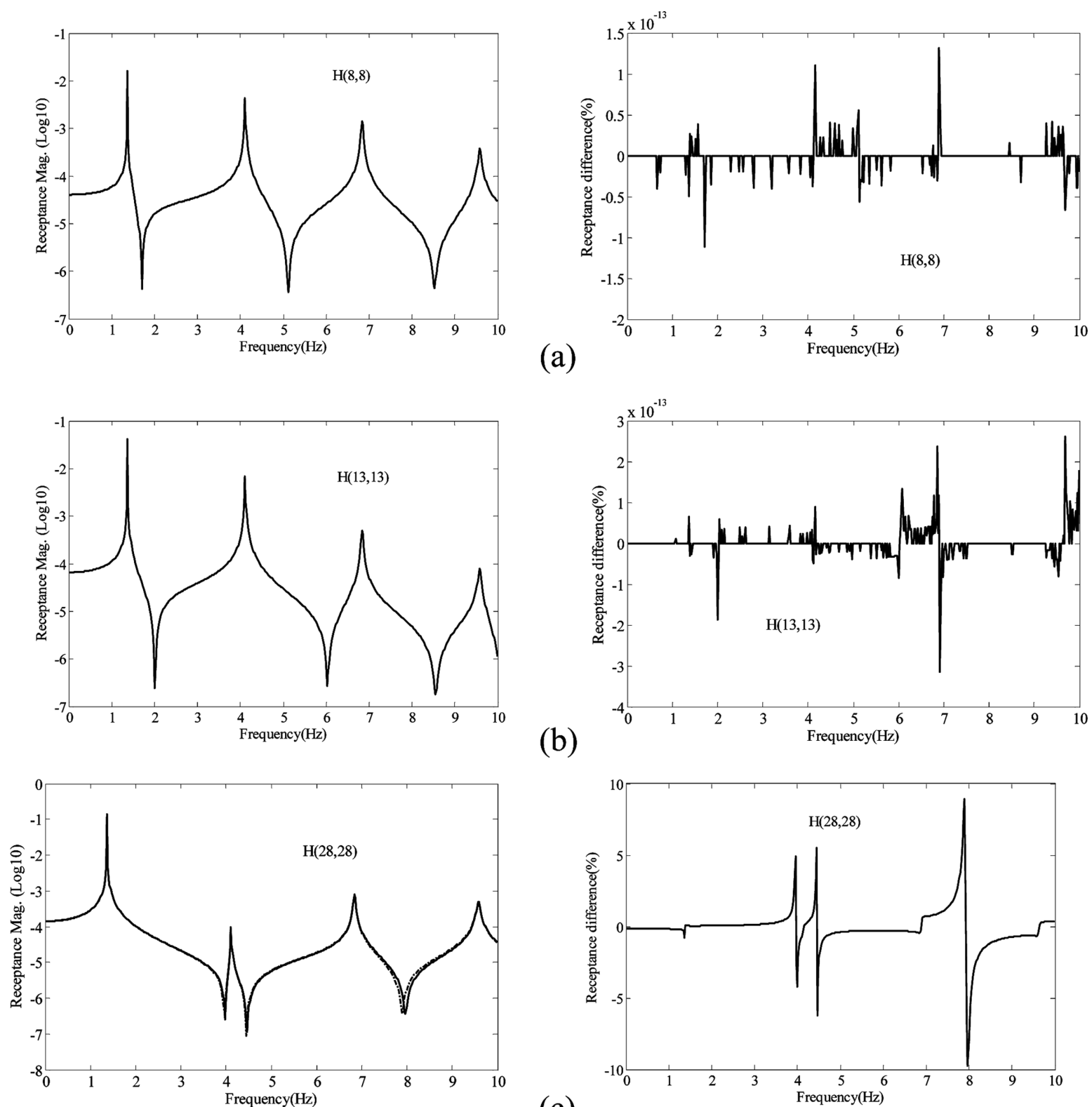

(b)

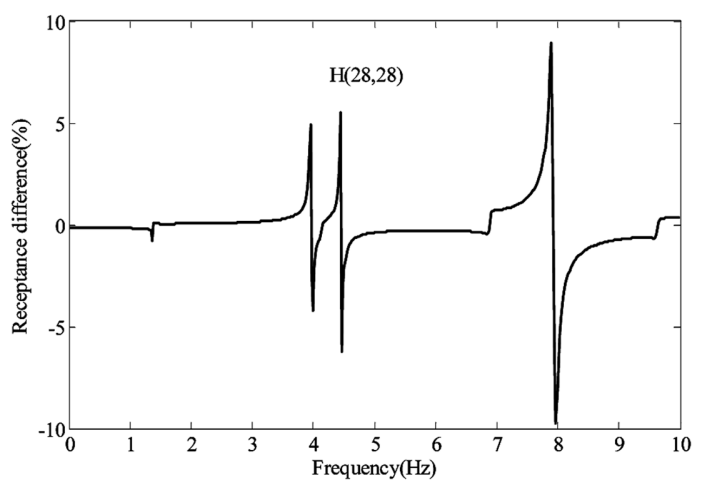

(c)

Fig. 3 FRF curves: (a) $H_{8,8}$, (b) $H_{13,13}$, (c) $H_{28,28}$, (d) $H_{33,33}$, (e) $H_{37,37}$ and (f) $H_{37,38}$

parameter matrices identified by this study, Lee \& Kim's method and Fritzen's method. Each discrepancy value corresponds to the row and column in the parameter matrices. The parameter matrices were calculated using the FRF data corresponding to two frequencies of $10 \mathrm{~Hz}$ and $10.5 \mathrm{~Hz}$. It is found that all three methods similarly identify the parameter matrices and the discrepancy comes from the incomplete measured data.

Fig. 5 exhibits the diagonal components of the variations in the parameter matrices presented in Fig. 4. The discrepancy indicates the difference in the parameter matrices between the initial system 

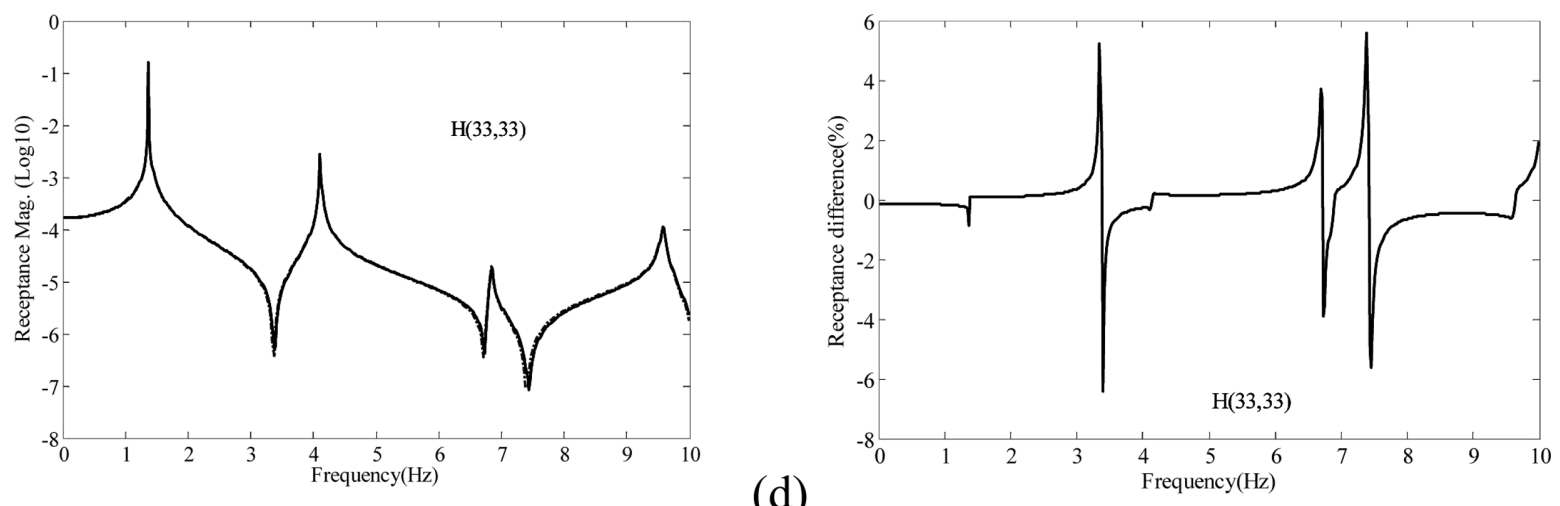

(d)
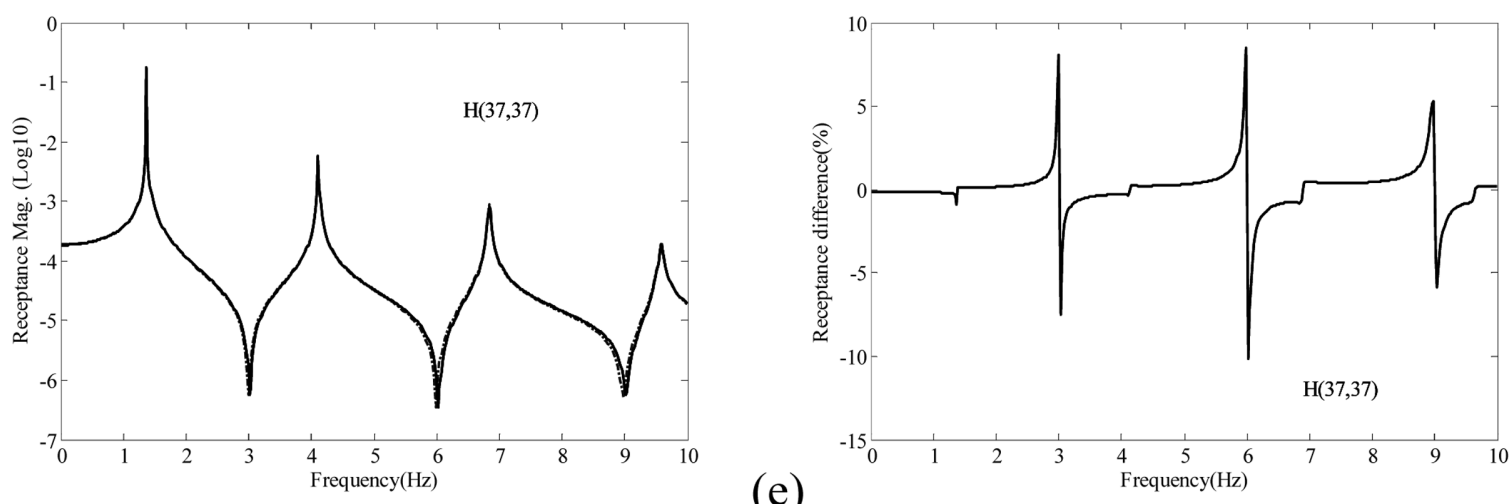

(e)
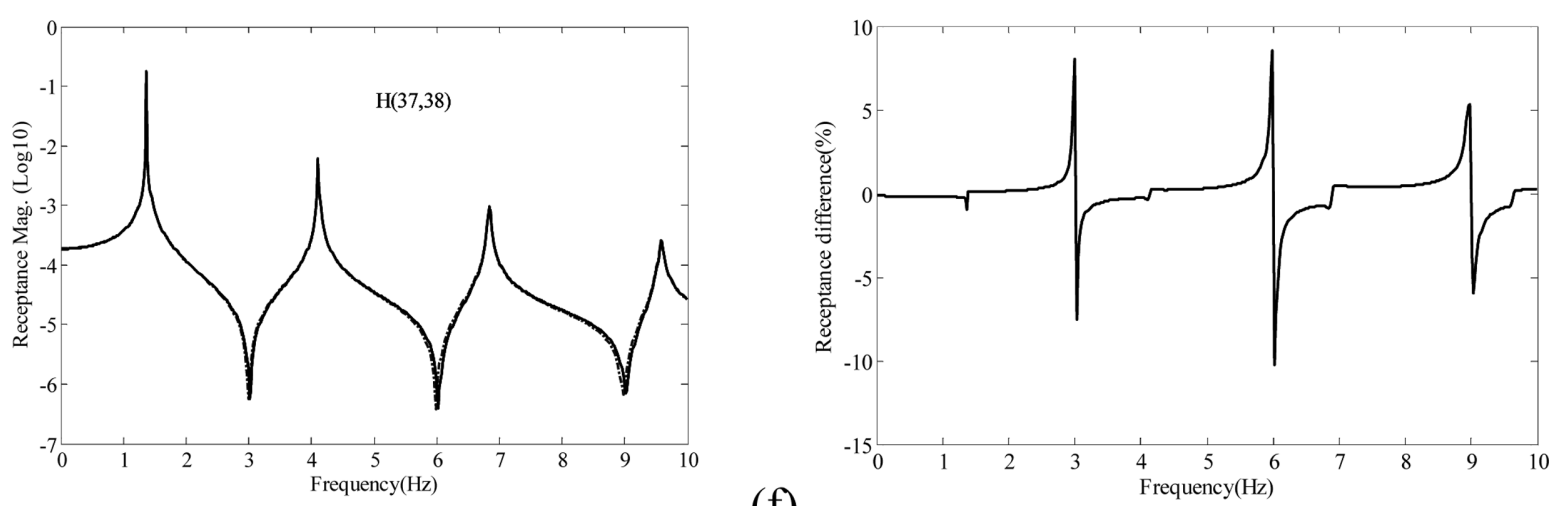

(f)

Fig. 3 (continued)

and the damaged system. It can be recognized from the plots that the damages exist in the region to represent the abrupt variation in the parameter values. Figs. 5(a), 5(d) and 5(g) represent the variation in the stiffness, damping and mass matrices based on the parameter identification method presented in this study, respectively. It is observed from the plots that the damage elements can be explicitly detected. Figs. 5(b), 5(e) and 5(h), and 5(c), 5(f) and 5(i) exhibit the variations in the stiffness, damping and mass matrices based on Lee and Kim's method and Fritzen's method, respectively. We can recognize the damage elements from the plots. And despite the assumption that the mass matrix is 


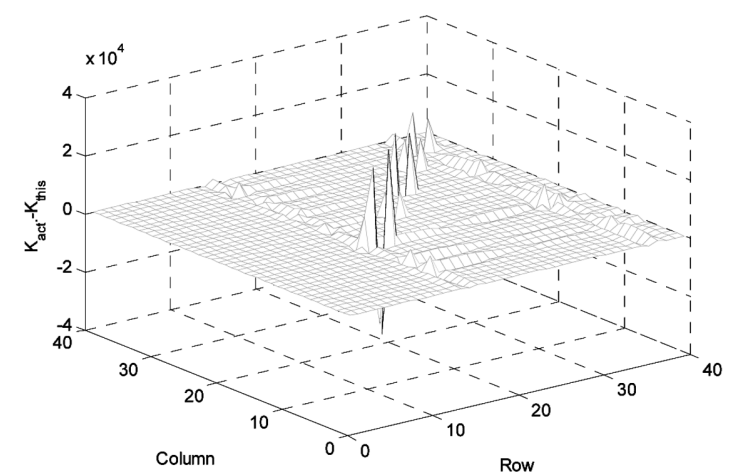

(a)

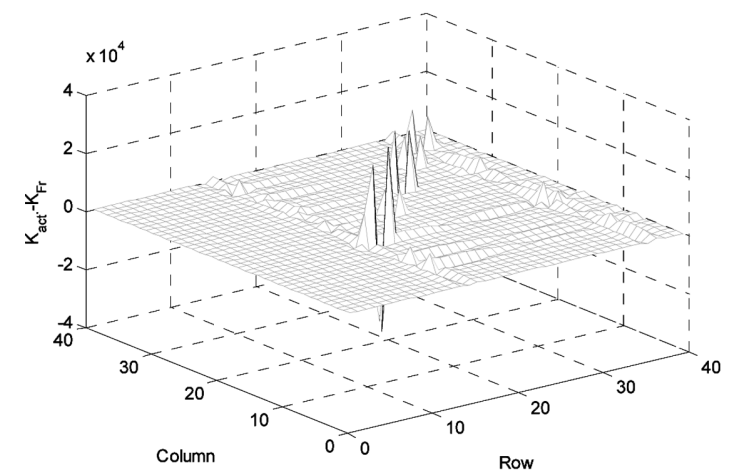

(c)

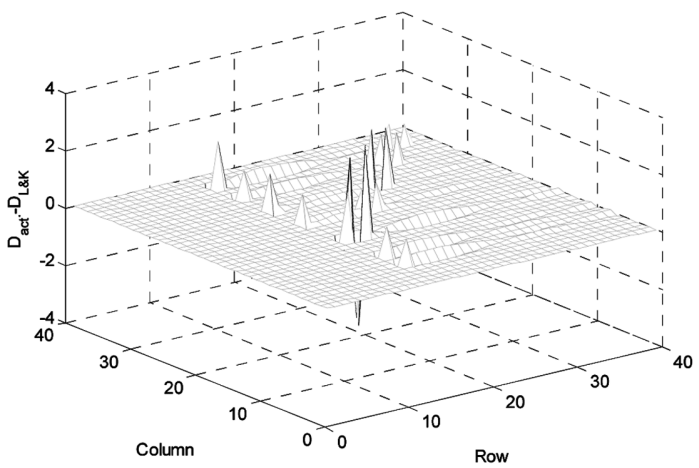

(e)

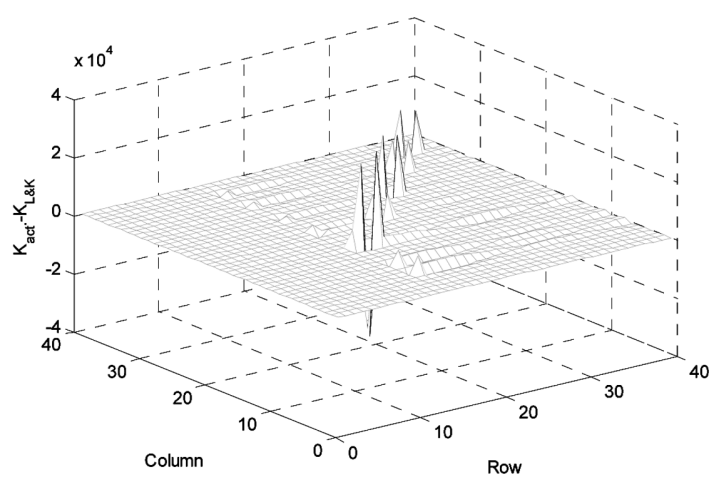

(b)

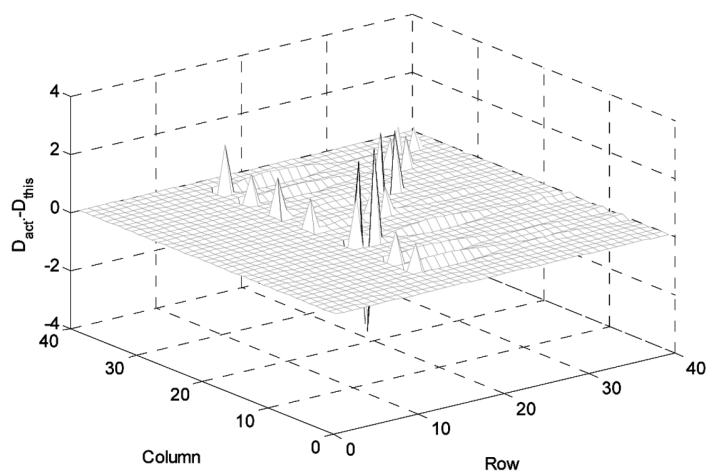

(d)

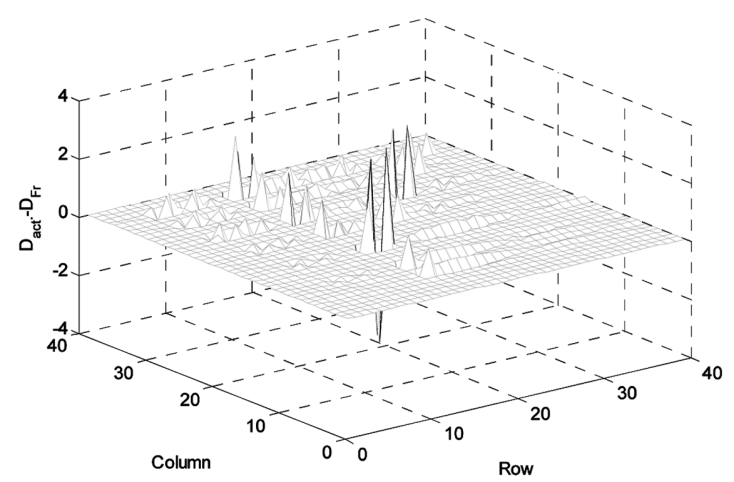

(f)

Fig. 4 Variations in the parameter matrices between the initial system and damaged system: (a) stiffness matrix by this study, (b) stiffness matrix by Lee and Kim's method, (c) stiffness matrix by Fritzen's method, (d) damping matrix by this study, (e) damping matrix by Lee and Kim's method and (f) damping matrix by Fritzen's method.

not changed, the change of the stiffness leads to the change of the damping and mass matrices. The damages also are detected by the mass variations. All three parameter identification methods can detect the damaged locations, but they exhibit small different numerical values of the parameter 


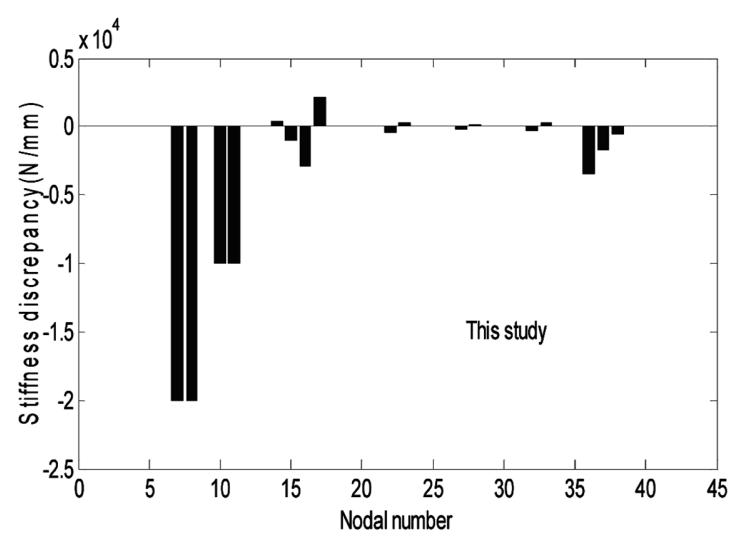

(a)

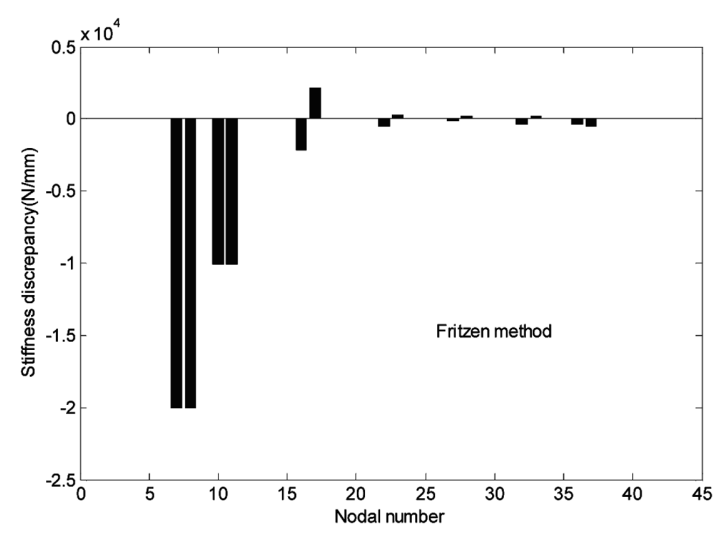

(c)

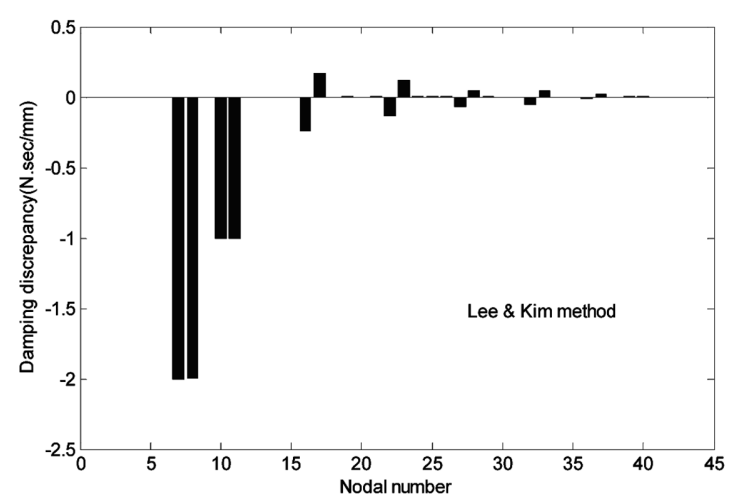

(e)

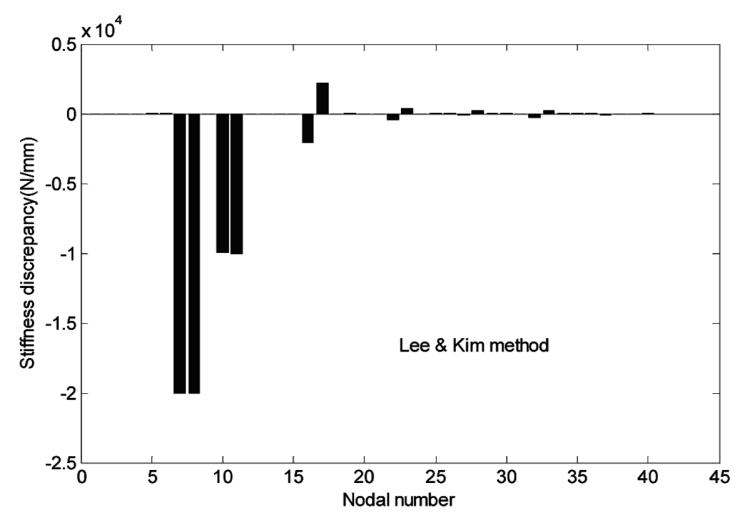

(b)

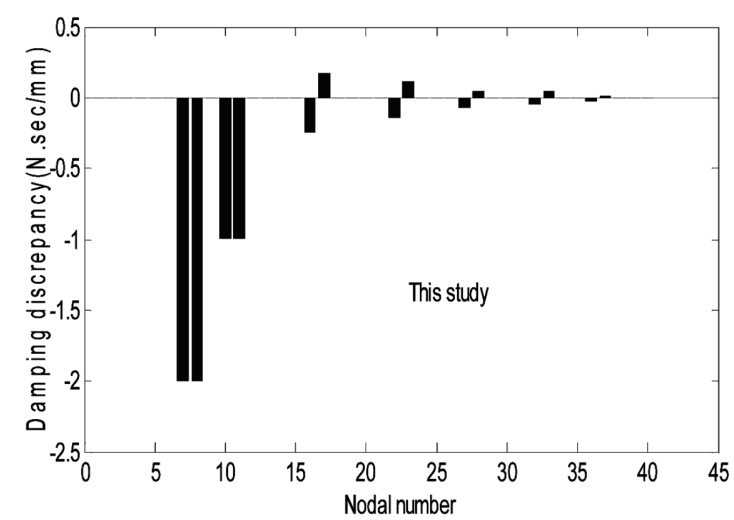

(d)

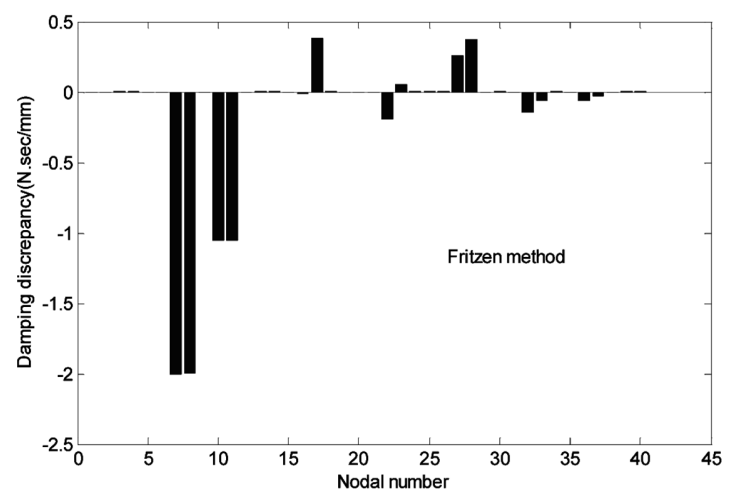

(f)

Fig. 5 Diagonal components in the variation of parameter matrices before and after damage: (a) stiffness variation by this study, (b) stiffness variation by Lee and Kim's method, (c) stiffness variation by Fritzen's method, (d) damping variation by this study, (e) damping variation by Lee and Kim's method, (f) damping variation by Fritzen's method, (g) mass variation by this study, (h) mass variation by Lee and Kim's method and (i) mass variation by Fritzen's method 


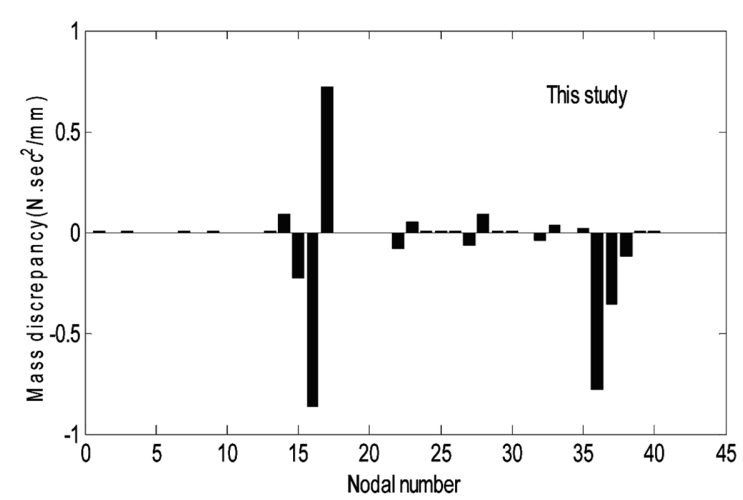

(g)

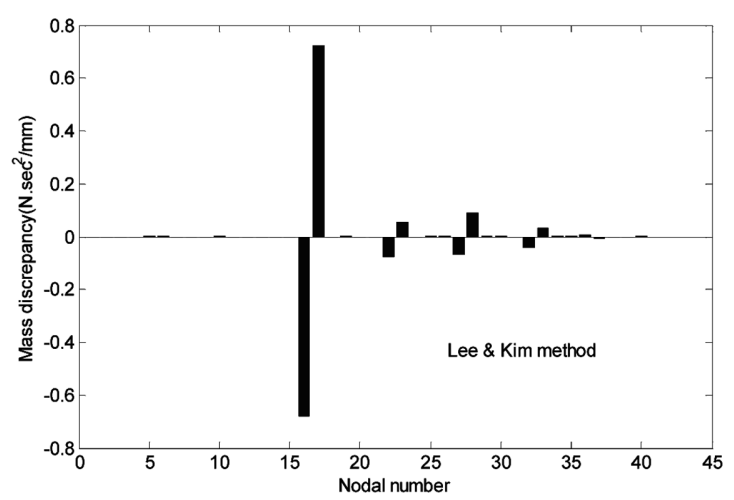

(h)

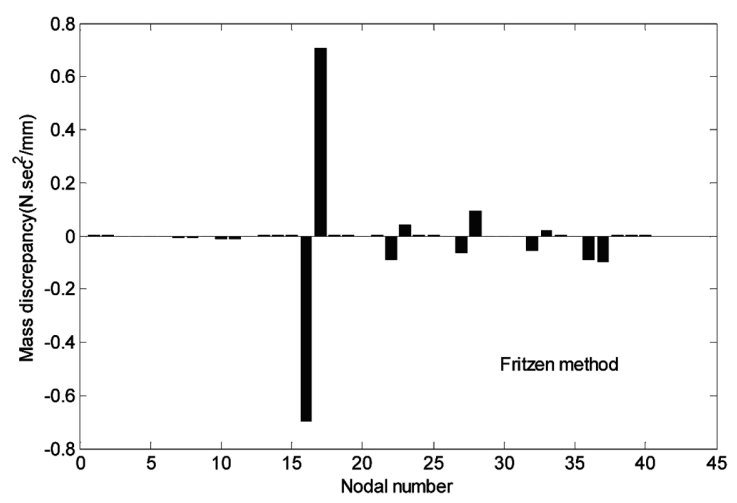

(i)

Fig. 5 (continued)

variations as shown in Fig. 5. The difference in the numerical values came from the calculation of the inverse of the entire FRF matrix expressed as measured complex values and the calculation of the Moore-Penrose inverse with respect to all three parameter matrices, unlike the proposed method and Lee and Kim's method. As exhibited in the application, it is concluded that the proposed expansion method and the parameter identification method provide the necessary information for damage detection.

\section{Conclusions}

Starting with incomplete measured FRF data, this study presented analytical methods to expand them to a full set of FRF matrix and to identify the parameter matrices from the estimated FRF matrix. It was investigated that the proposed parameter identification method does not require the inverse of the entire FRF matrix of experimentally measured complex values and the calculation of the Moore-Penrose inverse with respect to all three parameter matrices simultaneously. It can be concluded that the damage identification method provided in this study presents basic information for damage detection. 


\section{Acknowledgements}

This research was supported by Basic Science Research Program through the National Research Foundation of Korea (NRF) funded by the Ministry of Education, Science and Technology (no. 2009-0066266).

\section{References}

Avitabile, P. and O'Callahan, J. (2001), "Dynamic expansion of FRFs for the full FRF matrix", Proceedings of the $19_{\text {th }}$ IMAC.

Avitabile, P. and O'Callahan, J. (2003), "Frequency response function expansion for unmeasured translation and rotation dofs for impedance modeling applications", Mech. Syst. Signal Pr., 17(4), 723-745.

Cha, P.D. and Tuck Lee, J.P. (2000), "Updating structural system parameters using frequency response data", $J$. Eng. Mech-ASCE., 126(12), 1240-1246.

Eun, H.C., Lee, E.T. and Chung, H.S. (2004), "On the static analysis of constrained structural systems", Can. J. Civil Eng., 31(6), 1119-1122.

Ewins, D.J. (2000), "Adjustment or Updating of Models", Sâdhanâ, 25(3) 235-245.

Fanning, P.J. and Carden, E.P. (2004), "Experimentally validated added mass identification algorithm based on frequency response functions", J. Eng. Mech-ASCE., 130(9), 1045-1051.

Friswell, M.I. and Penny, J.E.T. (1990), "Updating model parameters from frequency domain data via reduced order models", Mech. Syst. Signal Pr., 4(5), 377-391.

Fritzen, C.P. (1986), "Identification of mass, and stiffness matrices of mechanical systems", J. Vib. Acoust., 108, 9-16.

Guyan, J. (1965), "Reduction of stiffness and mass matrices", AIAA J., 3(2), 380.

Kenneth, F.A. and Francois, H. (2000), "Dynamic mode shape expansion using mass orthogonality constraints", Proceedings of the $18_{\text {th }}$ IMAC.

Kwon, K.S. and Lin R.M. (2004), "Frequency selection method for FRF-based model updating", J. Sound. Vib., 278(1-2), 285-306.

Lee, J.H. and Kim, J. (2001), "Identification of damping matrices from measured frequency response functions", J. Sound. Vib., 240(3), 545-565.

Lee, U. and Shin, J. (2002), "A frequency response function-based structural damage identification method", Comput. Struct., 80(2), 117-132.

Lin, R.M. and Zju, J. (2006), "Model updating of damped structures using FRF data", Mech. Syst. Signal Pro., 20(8), 2200-2218.

Mottershead, J.E. and Stanway, R. (1986), "Identification of structural vibration parameters by using a frequency domain filter", J. Sound Vib., 109(3), 495-506.

O'Callahan, J. (1989) “A Procedure for an Improved Reduced System (IRS) Model”, Proceedings of the 7 th IMAC.

O’Callahan J, Avitabile, P.P. and Riemer, R. (1989), "System Equivalent Reduction Expansion Process (SEREP)", Proceedings of the 7 th $I M A C$.

Phane, A.S. and Seshia, A.A. (2004), "Identification of anisoelasticity and nonproportional damping in MEMS gyroscopes", NSTI-Nanotech, 2, 343-346.

Phani, A.S. and Woodhouse, J. (2007), "Viscous damping identification in linear vibration", J. Sound Vib., 303(3-5), 475-500 


\section{Appendix}

The response of an initial structure in the frequency domain, described by $\hat{\mathbf{U}}(\Omega)$, to an external excitation, described by $\mathbf{F}(\Omega)$, is given by

$$
\hat{\mathbf{U}}(\Omega)=\hat{\mathbf{H}}(\Omega) \mathbf{F}(\Omega)
$$

where $\hat{\mathbf{H}}(\Omega)=\left(\mathbf{K}-\Omega^{2} \mathbf{M}+i \Omega \mathbf{C}\right)^{-1}$ is the FRF matrix of the initial structure, whose elements can be receptances. Eq. (A.1) expresses the dynamic equation at a certain frequency $\Omega$.

Assume that the modal displacements in the frequency domain are measured as

$$
\mathbf{A} \mathbf{U}(\Omega)=\mathbf{b}
$$

in which A denotes a coefficient matrix, b represents the measured data and Eq. (A.2) represents constraints to govern the dynamic responses. Minimizing a quadratic form by the difference between the analytical response vector of Eq. (A.1) and the actual response vector of Eq. (A.2), the estimated response vector in the satisfaction of the constraint conditions of Eq. (A.2) can be obtained as

$$
\mathbf{U}=\hat{\mathbf{U}}+\hat{\mathbf{D}}^{-1 / 2}\left(\mathbf{A} \hat{\mathbf{D}}^{-1 / 2}\right)^{+}(\mathbf{b}-\mathbf{A} \hat{\mathbf{U}})
$$

where $\hat{\mathbf{U}}=\hat{\mathbf{D}}^{-1} \mathbf{F}$ and $\hat{\mathbf{H}}^{-1}=\hat{\mathbf{D}}$. Eq. (A.3) represents the dynamic equation for describing the constrained motion. 